\title{
A Hybrid Genetic Algorithm-Interior Point Method for Optimal Reactive Power Flow
}

\author{
Wei Yan, Fang Liu, C. Y. Chung, Member, IEEE, and K. P. Wong, Fellow, IEEE
}

\begin{abstract}
By integrating a genetic algorithm (GA) with a nonlinear interior point method (IPM), a novel hybrid method for the optimal reactive power flow (ORPF) problem is proposed in this paper. The proposed method can be mainly divided into two parts. The first part is to solve the ORPF with the IPM by relaxing the discrete variables. The second part is to decompose the original ORPF into two sub-problems: continuous optimization and discrete optimization. The GA is used to solve the discrete optimization with the continuous variables being fixed, whereas the IPM solves the continuous optimization with the discrete variables being constant. The optimal solution can be obtained by solving the two sub-problems alternately. A dynamic adjustment strategy is also proposed to make the GA and the IPM to complement each other and to enhance the efficiency of the hybrid proposed method. Numerical simulations on the IEEE 30-bus, IEEE 118-bus and Chongqing 161-bus test systems illustrate that the proposed hybrid method is efficient for the ORPF problem.
\end{abstract}

Index Terms-Genetic algorithm (GA), interior point method (IPM), nonlinear programming, optimal reactive power flow (ORPF).

\section{INTRODUCTION}

$\mathbf{T}$ HE MAIN purpose of optimal reactive power flow (ORPF) is to minimize the total power losses of the network while maintaining the voltage profile of the network in an acceptable range. The control variables of the study include voltages of generators, tap ratios of transformers, and reactive power generation of var sources (banks of capacitors and reactors). Since the control variables include both discrete variables (var sources and transformer tap ratios) and continuous variables (generator voltages), the ORPF is inherently a mixed-integer nonlinear programming (MINLP) problem [1].

Genetic algorithm (GA) is one kind of global optimization techniques with the advantage of dealing with the integer variables. Interior point method (IPM) offers fast convergence to

Manuscript received September 15, 2005; revised February 11, 2006. This work was supported in part by The Hong Kong Polytechnic University under Central Research Grant A-PF87, in part by The National Natural Science Foundation of China under Contract 50577073, in part by Chongqing University, and in part by Chongqing Electric Power Corporation. Paper no. TPWRS-005842005.

W. Yan is with the Key Laboratory of High Voltage Engineering and Electrical New Technology in Ministry of Education, Chongqing 400030, China (e-mail: cquyanwei@21cn.com).

F. Liu is with the Electrical Power Department, Electrical Engineering College, Chongqing University, Chongqing 400044, China (e-mail: fangfangr_liu@hotmail.com).

C. Y. Chung and K. P. Wong are with the Computational Intelligence Applications Research Laboratory (CIARLab), Department of Electrical Engineering, The Hong Kong Polytechnic University, Kowloon, Hong Kong (e-mail: eecychun@polyu.edu.hk; eekpwong@ polyu.edu.hk).

Digital Object Identifier 10.1109/TPWRS.2006.879262 solve large-scale nonlinear program problem. Both of them have been successfully applied to solving ORPF problem [2]-[7], but the difficulty of IPM in dealing with discrete variables and the tardiness of GA in searching optimal solution virtually remain unsolved.

The GA is a general evolution concept-based methodology, and its developing trend is to be combined with other algorithms [8], [9]. In [8], a combined methodology is presented, which consists of a successive linear programming (SLP) and a simple genetic algorithm (SGA). First, an initial solution is obtained by relaxing the discrete nature of all variables, and then a mixed linear integer problem is formulated and solved using the SGA and the SLP. The SGA deals with the optimization of discrete variables. The SLP works as a support technique for SGA, which provides the final system operating state by adjusting the existing continuous variables and finding the fitness function for each individual of the genetic algorithm population. A hybrid formulation using a GA combined with an IPM (HGI) has been reported in [9]. Similarly, the IPM is embedded in the process of GA to replace the load flow calculation and is employed to find the fitness for each candidate solution. Although the hybrid method is more efficient than the SGA, the execution time is still considerable. Indeed, it is unnecessary to use the IPM to find the fitness for each candidate solution of the GA. Besides, the randomicity of discrete variables may make the final system operating state become infeasible, no matter what the continuous variables are. In this case, the IPM is non-convergent. In order to integrate the GA with the IPM in a more effective way, a novel hybrid algorithm is therefore proposed in this paper.

This paper is organized as follows. A brief review of the ORPF problem formulation, the IPM, and the GA methodology is given in Section II-IV, respectively. In Section V, the novel hybrid method integrating the GA and the IPM (NHGI) is proposed, and a dynamic adjustment strategy is also introduced to improve the efficiency of the proposed method. In Section VI, the numerical simulation results of the NHGI on IEEE 30-bus, IEEE 118-bus, and Chongqing 161-bus test systems are shown and compared with that of HGI in [9]. Finally, Section VII concludes this paper.

\section{PROBlem Formulation}

The formulation of the ORPF problem can be expressed as the following MINLP problem:

$$
\begin{array}{ll}
\min & f(\boldsymbol{z}) \\
\text { s.t. } & \boldsymbol{g}(\boldsymbol{z})=0 \\
& \boldsymbol{z}_{\min } \leq \boldsymbol{z} \leq \boldsymbol{z}_{\max }
\end{array}
$$


where the objective function $f(\cdot)$ is the total active power losses; $g(\cdot)$ is the nonlinear vectors function representing power flow equations; $\boldsymbol{z}=\left[\boldsymbol{x}, \boldsymbol{u}_{C}, \boldsymbol{u}_{\boldsymbol{D}}\right]$ is the vector of decision variables including the vector of state variables $\boldsymbol{x}$ (voltage magnitudes and angles of the load buses and injected reactive powers of the generators), the vector of continuous control variables $\boldsymbol{u}_{\boldsymbol{C}}$ (generator voltages magnitude $\boldsymbol{V}_{G}$ ), and the vector of discrete control variables $\boldsymbol{u}_{\boldsymbol{D}}$ (reactive power $\boldsymbol{Q}_{C}$ of the shunt capacitor/reactor and transformer tap ratios $\boldsymbol{T}$ ); and $\boldsymbol{z}_{\min }$ and $\boldsymbol{z}_{\max }$ are the vectors modeling operational limits on state and control variables.

\section{INTERIOR POINT METHOD}

The ORPF problem in (1) can be solved by a nonlinear IPM based on a logarithmic barrier primal-dual algorithm defined in [5] and [6]. In this method, all the decision variables, $\boldsymbol{z}$ in (1), are first assumed to be continuous. Besides the slack variables, the Lagrange multipliers are introduced to deal with the inequality and equality constraints, and the logarithmic barrier functions are used to guarantee the non-negativity conditions of the slack variables. The ORPF problem can be transformed into the sub-problem of the following Lagrange function without the constraints:

$$
\begin{array}{r}
L(\boldsymbol{z}, \boldsymbol{y}, \boldsymbol{l}, \boldsymbol{u}, \boldsymbol{v}, \boldsymbol{w})=f(\boldsymbol{z})-\boldsymbol{v}^{T}\left(\boldsymbol{z}-\boldsymbol{l}-\boldsymbol{z}_{\min }\right)-\boldsymbol{y}^{T} \boldsymbol{g}(\boldsymbol{z}) \\
+\boldsymbol{w}^{T}\left(\boldsymbol{z}+\boldsymbol{u}-\boldsymbol{z}_{\max }\right)-\mu \sum_{i}\left(\ln l_{i}+\ln u_{i}\right)
\end{array}
$$

where $\boldsymbol{u}$ and $\boldsymbol{l}$ are the slack variables ( $\boldsymbol{u} \geq 0, \boldsymbol{l} \geq 0$ ); $\boldsymbol{y}, \boldsymbol{v}$, and $\boldsymbol{w}$ are the Lagrange multipliers $(\boldsymbol{v} \geq 0, \boldsymbol{w} \geq 0)$; and $\mu$ is the barrier parameter $(\mu>0)$.

Based on the Karush-Kuhn-Tucker (KKT) first-order conditions of the sub-problem, a set of nonlinear algebraic equations is formed and then solved by the Newton-Raphson algorithm. The iteration procedure of the IPM is stopped when the mismatches of KKT conditions are sufficiently small or less than the specified tolerance $(\varepsilon)$ as shown in the following:

$$
\begin{aligned}
\left\|L_{\boldsymbol{z}}\right\| & =\left\|\nabla f(\boldsymbol{z})-\nabla \boldsymbol{g}^{T}(\boldsymbol{z}) \boldsymbol{y}-\boldsymbol{v}+\boldsymbol{w}\right\|<\varepsilon \\
\left\|L_{\boldsymbol{y}}\right\| & =\|\boldsymbol{g}(\boldsymbol{z})\|<\varepsilon \\
\left\|L_{v}\right\| & =\left\|\boldsymbol{z}-\boldsymbol{l}-\boldsymbol{z}_{\min }\right\|<\varepsilon \\
\left\|L_{\boldsymbol{w}}\right\| & =\left\|\boldsymbol{z}+\boldsymbol{u}-\boldsymbol{z}_{\max }\right\|<\varepsilon \\
G a p & =\boldsymbol{v}^{T} \boldsymbol{l}+\boldsymbol{w}^{T} \boldsymbol{u}<\varepsilon .
\end{aligned}
$$

In the primal-dual theory, $\boldsymbol{z}, \boldsymbol{l}$, and $\boldsymbol{u}$ are the primal variables; $\boldsymbol{y}, \boldsymbol{v}$, and $\boldsymbol{w}$ are the dual variables; (3) is the dual feasible condition; (4)-(6) are the primal feasible conditions; and (7) is the complementary slackness condition. Therefore, the optimal solution fulfills the stopping criteria in (3)-(7), while the feasible solution satisfies the stopping criteria in (4)-(6).

\section{Genetic AlgORITHM}

Genetic algorithm (GA) is an optimization algorithm based on the mechanics of natural selection and genetics. The approach is based on Darwin's survival of the fittest hypothesis. In the GA, candidate solutions to the given problem are analogous to individuals in a population. Each individual is encoded as a string, called chromosome. New candidate solutions are produced from parent chromosomes by the crossover operator. The mutation operator is then applied to the population. The quality of each individual is evaluated and rated by the so-called fitness function. Similar to the natural selection mechanism in the biological system, the fitter individuals have more chance to pass on information to the next generation. When a chromosome with the desired fitness is formed, it will be taken as the optimal solution, and the optimization process is terminated. Otherwise, the process is repeated until the maximum number of generations is reached and the fittest chromosome so far formed is taken to be the optimal solution. In this paper, the simple genetic algorithm (SGA) in [3] is implemented with some modifications. The different components of GA are described as follows.

1) Chromosomes: Since the transformer tap ratios and shunt capacitor/reactor capacitors are all discrete control variables, they can be encoded as integer variables. When a discrete control variable $u_{D}$ is expressed by (8), the integer-encoded gene of the control variable can be represented by the integer $N$. By this encoding method, the length of the chromosome is equal to the number of control variables, and each gene represents a control parameter of ORPF

$$
u_{D}=\left\{u_{D \min }+N \times \Delta u_{D} \mid N \in\left\{0,1, \cdots, N_{\max }\right\}\right\}
$$

where $u_{D \min }$ and $\Delta u_{D}$ are the lower limit and the stepsize of the discrete control variable, respectively. $N_{\max }$ is the maximum number of control steps for the corresponding variable.

2) Fitness Function: The objective of ORPF is to minimize the total active power loss. GA is designed to maximize the fitness, which is a measure of the quality of each candidate solution. Therefore, a transformation is needed to convert the objective of ORPF to an appropriate fitness function. The control variable constraints of ORPF are automatically satisfied by the encoding scheme, while the state variable constraints are needed to be included in the GA fitness function by penalty terms. In this paper, the fitness function is formed as follows:

$$
\begin{aligned}
F & =-f\left(\boldsymbol{u}_{\boldsymbol{D}}\right)-\sum_{j} c_{j} \text { Pen }_{j} \\
\text { Pen }_{j} & = \begin{cases}x_{j \min }-x_{j}, & \text { if } x_{j}<x_{j \min } \\
x_{j}-x_{j \max }, & \text { if } x_{j}>x_{j \max } \\
0, & \text { otherwise }\end{cases}
\end{aligned}
$$

where $f\left(\boldsymbol{u}_{\boldsymbol{D}}\right)$ is the objective function with control variables $\boldsymbol{u}_{\boldsymbol{D}} ; x_{j}$ is the $j$ th state variable; $x_{j \min }$ and $x_{j \max }$ are the lower and upper limits of $x_{j} ; c_{j}$ is a penalty factor for $x_{j} ; P e n_{j}$ is the violation value of $x_{j}$; and $\sum_{j} c_{j} P e n_{j}$ is the penalty function for state variable constraints.

With every candidate solution, the chromosome is decoded to determine the control variables $\boldsymbol{u}_{\boldsymbol{D}}$, and the corresponding state variables $\boldsymbol{x}$ are computed by the power 
flow calculation. The fitness function of this candidate solution can then be calculated by (9).

3) Selection operation: Selection is a procedure to guarantee that individuals with higher fitness values have a higher probability of contributing new offspring to the next generation. The selection rule used in this paper is the tournament selection [13].

4) Crossover operation: Crossover produces new chromosomes by the combination of parent individuals. In this paper, the uniform crossover method [4] is adopted with a crossover rate of 0.8 .

5) Mutation operation: Mutation is responsible for the injection of new information. For integer-coded chromosome, the mutation operation is defined as

$$
N_{m u t}= \begin{cases}N_{i n i}+R A N D\left\langle N_{\max }-N_{i n i}\right\rangle & (\gamma=1) \\ N_{i n i}-R A N D\left\langle N_{i n i}\right\rangle & (\gamma=0)\end{cases}
$$

where $\gamma$ is a random bit; $N_{m u t}$ is the gene after mutation; $N_{i n i}$ is the gene before mutation; and $R A N D\langle N\rangle$ returns a random integer in the range of $[0, N]$. In this paper, the mutation operator is applied with a probability of 0.03 to every gene of the chromosome.

\section{HYBRID ALGORITHM FOR ORPF}

\section{A. Novel Hybrid Method}

A hybrid method integrating the GA and the IPM is introduced in this section. This method can be mainly divided into two parts. The first part employs the IPM to solve the ORPF problem approximated as a continuous problem. Then the optimal solution obtained is rounded off, in which continuous values for discrete variables are discretised, and used as the initial population of GA. The second part is to combine the GA with the IPM to solve the ORPF for the final optimal solution. In this part, the original ORPF problem is decomposed into two sub-problems. One is the continuous optimization sub-problem, in which the discrete control variables including var sources and transformer tap ratios in ORPF, are kept constant. This sub-problem is solved by IPM with the variables in (1) and (2) expressed as $\boldsymbol{z}_{C}\left(=\left[\boldsymbol{x}, \boldsymbol{V}_{G}\right]\right)$. The other one is the discrete optimization sub-problem in which the continuous control variables, including generator voltages in ORPF, are kept constant. GA is used to solve this sub-problem with the variables in (1) and (9) expressed as $\boldsymbol{z}_{D}\left(=\left[\boldsymbol{x}, \boldsymbol{Q}_{C}, \boldsymbol{T}\right]\right)$.

The procedure of the proposed hybrid algorithm is summarized as follows.

Step 1) Solve the ORPF problem by IPM with relaxing the discrete variables to obtain the initial solution $\boldsymbol{V}_{G}{ }^{(0)}, \boldsymbol{Q}_{C}{ }^{(0)}$ and $\boldsymbol{T}^{(0)}$

Step 2) Set iteration count $k=1$.

Step 3) Determine the optimal $\boldsymbol{Q}_{C}{ }^{(k)}$ and $\boldsymbol{T}^{(k)}$ by solving the discrete optimization sub-problem using GA with keeping $\boldsymbol{V}_{G}{ }^{(k)}=\boldsymbol{V}_{G}{ }^{(k-1)}$. At the same time, the last solution $\left[\boldsymbol{V}_{G}{ }^{(k-1)}, \boldsymbol{Q}_{C}{ }^{(\dot{k-1)}}, \boldsymbol{T}^{(k-1)}\right]$ is put into the original population as one individual, and the best individual in each generation is kept in the next population in the whole GA iteration.
Step 4) Determine the optimal $\boldsymbol{V}_{G}{ }^{(k)}$ by solving the continuous optimization sub-problem using IPM with the values of ${Q_{C}}^{(k)}$ and $\boldsymbol{T}^{(k)}$ obtained in step 3 constant. In the same way, the solution of GA is used as starting point of IPM.

Step 5) If the convergence criterion is satisfied, terminate the calculation. If not, set $k=k+1$ and then go to Step 3.

\section{B. Dynamic Adjustment Strategy}

To improve the efficiency of the proposed hybrid method, the following dynamic adjustment strategies are proposed.

1) Adjustment of Stopping Criterion in IPM: In Step 1 of the proposed hybrid method, the IPM with the "optimal criterion" given by (3)-(7) may result in oscillation for solving large-size ORPF, while the IPM with the "feasible criterion" given by (4)-(6) is easier to converge with high speed. Besides, the solution obtained is still continuous, even if using the optimal criterion, and when the discrete control variables are rounded off to discrete values, the performance of the solution will be inevitably deteriorated. Thus, it is unnecessary to use the IPM with the optimal criterion.

On the contrary, in Step 4, the continuous optimization subproblem should be solved using IPM with the optimal criterion, because there is no rounding problem and the dimension of this sub-problem with only the continuous control variables is relatively low.

Thus, in Step 1, IPM is used with the feasible criterion, and in Step 4, it is used with the optimal criterion.

2) Adjustment of Initial Population, Search Area and Stopping Condition in GA: In order to take the full advantage of the solution of IPM in Steps 1 and 4, the initial population, the search area, and stopping condition of GA in Step 3 are regulated with different iteration count $k$.

When $k=1$, all the initial individuals are created randomly within the range of two step sizes around the values of discrete control variables obtained by IPM in Step 1, and the search area in the whole genetic iteration process is also within this range. The stopping condition is when a feasible individual is found. This process is here called the "feasible adjusting strategy."

When $k>1$, all the initial individuals except one (obtained by IPM from Step 4) are produced randomly within the operational limits of discrete control variables given by (1), and the search area in the whole genetic iteration process is within this operational limits. The stopping condition is when the maximum number of iteration is reached or the optimal individual keeps unchanged for several generations. This process is here called the "optimal adjusting strategy."

There are two main reasons for the adjustments above. First, it is easy and quick, in most cases, to find a feasible individual around the solution obtained by IPM from Step 1, which is just like most applications of IPM to ORPF for getting the final discrete result by the rounding off process. Mass numerical tests demonstrate that if the feasible adjusting strategy is used, it only takes one or two genetic iterations of GA to find a feasible solution. The main purpose of using the optimal adjusting strategy is to provide GA sufficiently large search space to search the global optimal solution stochastically. 
TABLE I

BASIC INFORMATION FOR TEST SYSTEMS

\begin{tabular}{cccr}
\hline \hline Name & $\begin{array}{c}\text { Number of } \\
\text { bus / branch }\end{array}$ & $\begin{array}{c}\text { Number of control } \\
\text { variables } \\
\left(\boldsymbol{V}_{G} / \boldsymbol{Q}_{C} / \boldsymbol{T}\right)\end{array}$ & PL (pu) \\
\hline IEEE 30 & $30 / 41$ & $6 / 9 / 4$ & 0.0739 \\
IEEE 118 & $118 / 186$ & $54 / 14 / 9$ & 1.3359 \\
Chongqing 161 & $161 / 212$ & $8 / 44 / 44$ & 0.9648 \\
\hline \hline
\end{tabular}

\section{SimULATION RESULtS}

\section{A. Test Systems}

The test systems include the IEEE 30-bus and IEEE 118-bus systems, and a real power system in Chongqing, China. The Chongqing system has two equivalent electrical source buses, seven generators that are located in three power plants, three $500-\mathrm{kV}$ substations with five three-winding off-load tap changing transformers, $33220-\mathrm{kV}$ substations with 44 three-winding tap changing under load transformers, 44 shunt capacitor/reactor elements, and $3808 \mathrm{MW}$ load. The MVA base is 100 .

Table I shows the basic information for each test system and the initial active power losses (PL) in p.u. The programs implementing the proposed algorithms in Section $\mathrm{V}$ are coded in MATLAB and executed on a Pentium IV 2.0-GHz processor.

\section{B. Comparison of IPM, GA, and the Hybrid Algorithms}

In this paper, five methods, which are listed in Table II, were tested: (1) GA (from [12]) alone, (2) IPM (with optimal criterion from [6]) alone, (3) IPM as in (2) but continuous values for discrete variables are rounded off after convergence (IPM_D). (4) hybrid method (HGI) combined with IPM and GA from [9], and (5) proposed novel hybrid method (NHGI) in Section V. For the GA in Method 1 or GA of HGI and NHGI in Methods 4 and 5 , the population size is 20 , and the stopping criterion is that a feasible solution keeps unchanged for ten generations. For NHGI, one iteration calculation is finished only after both of the discrete and continuous sub-problems are solved. Therefore, in this example, the whole optimal calculation for NHGI stops when the solution keeps unchanged within two iterations.

The optimization results are listed in Table II. It is observed that GA and IPM with the rounding-off strategy cannot find feasible solutions. Method 2 using IPM produces the minimum active losses among other tests, but the solution is continuous and impractical. Only HGI and NHGI can produce feasible and practical solutions, but active losses from NHGI are less than those from HGI, and the CPU time taken by NHGI is much less than that taken by HGI. Comparing the CPU time, the active losses, and feasibility of solution, the result from NHGI is the best among the five methods. This paper shows that the proposed hybrid method has its superiority in optimality, and its ability in dealing with the discrete problem and the convergence speed is better than that of GA, IPM, and the hybrid method in [9].

For the IEEE 30-bus system, the optimization results of the control variables $\left(Q_{C}\right.$ and $\left.T\right)$ obtained by IPM, IPM-D, and NHGI have been shown in Table III. In general, the bus voltage
TABLE II

OPTIMIZATION RESULTS FOR TEST SYSTEMS

\begin{tabular}{cccccc}
\hline \hline $\begin{array}{c}\text { Test } \\
\text { System }\end{array}$ & Method & $\begin{array}{c}\text { CPU } \\
\text { time(s) }\end{array}$ & PL (pu) & $\begin{array}{c}\text { Reduction } \\
\text { of PL (\%) }\end{array}$ & $\begin{array}{c}\text { Iteration } \\
\text { counts }\end{array}$ \\
\hline \multirow{2}{*}{ IEEE } & GA* & 45 & 0.0738 & 1.27 & 400 \\
30 & IPM & 1.44 & 0.0666 & 9.88 & 10 \\
& HGI & - & 0.0667 & 9.74 & - \\
& NHGI & 6.7 & 0.0668 & 9.61 & 6 \\
\hline \multirow{4}{*}{$\begin{array}{c}\text { GE* } \\
\text { IEE }\end{array}$} & IPM & 111 & 0.0714 & 3.38 & 15 \\
& IPM_D* & - & 1.1450 & 14.29 & - \\
& HGI & 185 & 1.1681 & 12.56 & 12 \\
& NHGI & 18.6 & 1.1527 & 13.71 & 5 \\
\hline & GA* & 185 & 0.9465 & 1.9 & 400 \\
qing & IPM & 6.118 & 0.7793 & 19.23 & 16 \\
161 & IPM_D* & - & 0.7720 & 19.98 & - \\
& HGI & 286 & 0.8521 & 11.68 & 17 \\
& NHGI & 25.4 & 0.8423 & 12.7 & 5 \\
\hline \hline
\end{tabular}

Note: Symbol * means that some state variables in the solution violate their limits

TABLE III

OPTIMIZATION RESULTS FOR IEEE 30-BUS SYSTEM

\begin{tabular}{|c|c|c|c|c|}
\hline variables & $\begin{array}{c}\text { bus or branch } \\
\text { number }\end{array}$ & IPM & IPM-D & NHGI \\
\hline \multirow{9}{*}{$Q_{c}$} & 26 & 0.0222 & 0.03 & 0.03 \\
\hline & 28 & 0.0013 & 0 & 0.03 \\
\hline & 30 & 0.0264 & 0.03 & 0.03 \\
\hline & 12 & 0.0691 & 0.06 & 0.06 \\
\hline & 15 & 0.0404 & 0.06 & 0 \\
\hline & 18 & 0.0091 & 0 & 0 \\
\hline & 19 & 0.0382 & 0.05 & 0.05 \\
\hline & 21 & 0.107 & 0.1 & 0.1 \\
\hline & 24 & 0.0306 & 0.05 & 0.05 \\
\hline$\sum Q_{c}$ & & 0.3443 & 0.38 & 0.35 \\
\hline \multirow{4}{*}{$T$} & $6-9$ & 0.9772 & 0.975 & 0.9875 \\
\hline & $10-6$ & 0.9399 & 0.9375 & 0.95 \\
\hline & $4-12$ & 0.9712 & 0.975 & 0.9625 \\
\hline & $27-28$ & 1.0152 & 1.0125 & 1.0125 \\
\hline \multirow{4}{*}{$V_{b}$} & 3 & 1.05 & 1.0506 & 1.0494 \\
\hline & 9 & 1.0499 & 1.0516 & 1.0474 \\
\hline & 12 & 1.05 & 1.0503 & 1.05 \\
\hline & 27 & 1.0469 & 1.0503 & 1.05 \\
\hline
\end{tabular}

may violate its limits when the transformer tap ratio variable $(T)$ is rounded off. When the shunt reactive compensation variable $\left(Q_{c}\right)$ is rounded off, it may result in the limit violation of both bus voltage and reactive power output of generator. It can be observed from Table III that the total reactive power compensation $\left(\sum Q_{c}\right)$ in IPM is 0.3443 . After rounding off, it becomes 0.38 in IPM_D. Thus, most bus voltages are raised up because the total reactive power injection is increased. Voltage $\left(V_{b}\right)$ of the buses, including $3,9,12$, and 27 , have violated their upper voltage limits as shown in Table III because their original voltage magnitudes in IPM are very close their upper voltage limits (1.05 p.u.). This simulation result has demonstrated that 
TABLE IV

Statistical Data FOR DifFERENT Test Systems

\begin{tabular}{cccccc}
\hline \hline Name & Method & $\begin{array}{c}\text { Average } \\
\text { PL (pu) }\end{array}$ & $\begin{array}{c}\text { Standard } \\
\text { deviation }\end{array}$ & Max & Min \\
\hline IEEE & HGI & 0.0707 & 0.0021 & 0.0769 & 0.0669 \\
30 & NHGI & 0.0674 & 0.0006 & 0.0696 & 0.0666 \\
\hline IEEE & HGI & 1.1758 & 0.0065 & 1.1914 & 1.1574 \\
118 & NHGI & 1.1521 & 0.0023 & 1.1600 & 1.1471 \\
\hline Chong & HGI & 0.8512 & 0.0153 & 0.9049 & 0.8274 \\
qing & NHGI & 0.8324 & 0.0042 & 0.8529 & 0.8083 \\
\hline \hline
\end{tabular}

the traditional rounding-off strategy for the solution of ORPF obtained by IPM may not guarantee the feasibility of optimization result.

\section{Comparison of NHGI and HGI}

The proposed hybrid method (NHGI) and the hybrid algorithm (HGI) from [9] are basically different in the way of the integration of GA and IPM. To show the influence produced from the difference, 50 trials have been performed for NHGI and HGI methods in Section VI-B. The statistical results and the convergence are shown in Table IV and Fig. 1, respectively.

It is observed from Table IV that the optimal solutions obtained by both methods are feasible. The average, minimum, and maximum values of the active losses obtained by NHGI are better than those by HGI, and the standard deviation value from NHGI is relatively small. In Fig. 1, curve 1 is the result of NHGI, and curve 2 is the result of HGI. It is observed that the fitness values of the optimal individuals in the whole iteration process of NHGI are much better than that of HGI, especially in the first iteration calculation. Because the initial solution of NHGI is based on the result of IPM for ORPF with the discrete control variables being relaxed in Step 1, while the initial solution of HGI is created randomly.

The superiority of NHGI in the convergence speed can be guaranteed, even though it is unfair to compare the iteration counts for HGI and NHGI. This conclusion can be made by the following comparison: For NHGI in Section V-A, only one IPM calculation is performed after one GA process. For HGI in [9], IPM is embedded in the process of GA and is employed to find the fitness of each candidate solution and hence the number of IPM calculations is the number of the population size of the GA in this case. NHGI therefore requires much less IPM calculations than HGI. It is well known that the computational time for one candidate solution by the Fast Decoupled Method is much less than that by IPM. So the proposed NHGI method has noticeable advantage in speed over the HGI method.

The superiority of NHGI in the convergence speed and optimization of solution is obvious. It is noted that the proposed NHGI method is, however, still unable to guarantee the global optimal solution, which is a common weakness of the GA methods.

\section{Test for Effect of Dynamic Adjustment Strategy}

To compare the optimal criterion (IPM1) given in (3)-(7) with the feasible criterion (IPM2) given in (4)-(6) for the IPM

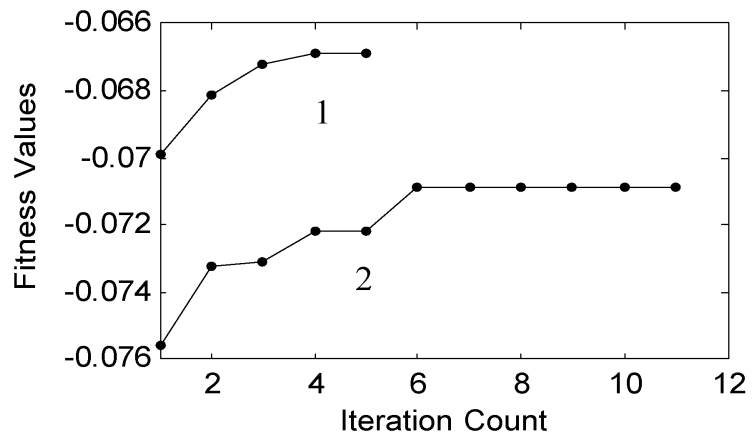

(a)

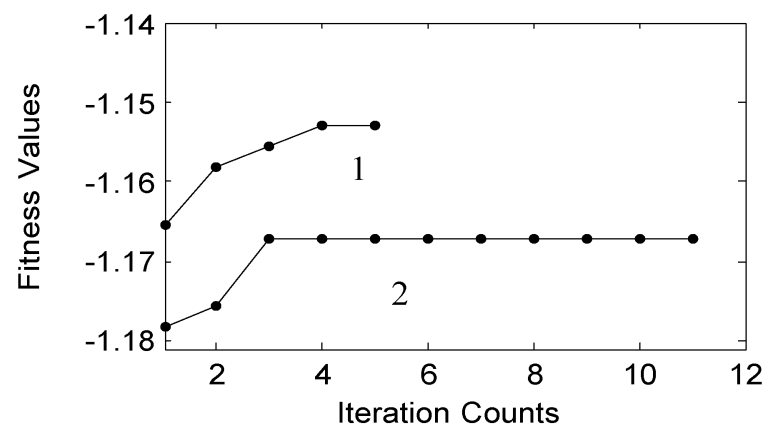

(b)

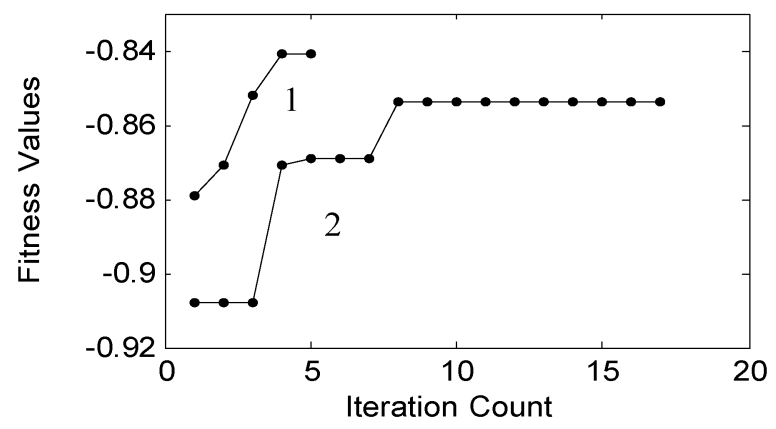

(c)

Fig. 1. Maximum fitness curves by HGI and NHGI methods. (a) IEEE 30-bus test system. (b) IEEE 118-bus test system. (c) Chongqing 161-bus test system.

method, the following tests are performed: 1) Solve the ORPF problem with the discrete control variables being relaxed by IPM1 and IPM2. 2) IPM1 and IPM2 as in (1) but continuous values for discrete variables are rounded off after convergence. 3) Produce 50 approximate solutions of (1) by using the random rounding-off method within the range of two step sizes around the discrete variables values obtained from (1). The results of these three tests are listed in Table V.

It is observed from Table $\mathrm{V}$ that the probability for IPM2 to find feasible solutions is much larger than that for IPM1, the CPU time taken by IPM2 is much less than that by IPM1, and the active losses solved by IPM2 are much larger than that by IPM1.

The above observations confirm the validity of the proposed strategy in Section V-B that in Step 1 of the proposed hybrid method, the feasible criterion is adopted for feasibility of the solutions and convergence speed, while in Step 4, the optimal criterion is used for optimality of the solutions. 
TABLE V

OPTIMAL RESULT FOR IPM WITH DIFFERENT CRITERIONS

\begin{tabular}{ccccccc}
\hline \hline Name & Method & CPU time & PL1 & PL2 & FNum & FPLav \\
\hline IEEE & IPM1 & $1.44 \mathrm{~s}$ & 0.0666 & $0.0667 *$ & 8 & 0.0674 \\
30 & IPM2 & $0.67 \mathrm{~s}$ & 0.0774 & 0.0781 & 50 & 0.0783 \\
\hline IEEE & IPM1 & $4.07 \mathrm{~s}$ & 1.1452 & $1.1450 *$ & 0 & - \\
118 & IPM2 & $0.94 \mathrm{~s}$ & 1.3179 & 1.3175 & 50 & 1.3186 \\
\hline $\begin{array}{c}\text { Chong } \\
\text { qing } \\
161\end{array}$ & IPM1 & $6.118 \mathrm{~s}$ & 0.7793 & $0.7720^{*}$ & 0 & - \\
\hline
\end{tabular}

In Table V, Symbol * means that the solution is infeasible. "CPU time" and "PL1" is for test (1). "PL2" is for test (2). "FNum" is the number of feasible solutions obtained from test (3). "FPLav" is the average active losses value of the feasible solutions obtained from test (3).

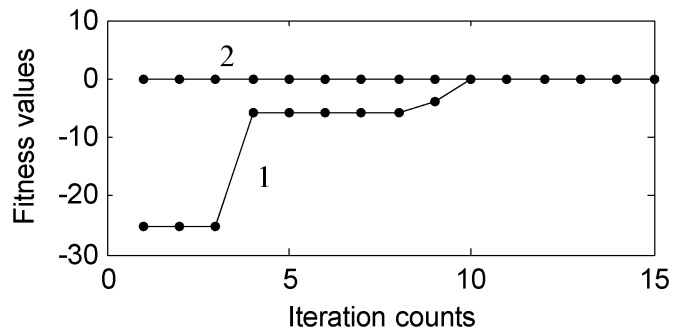

Fig. 2. Maximum fitness curves of the first discrete optimization in NHGI (with/without adjustment strategy) for IEEE 30-bus test system.

To verify the impact of the dynamic adjustment strategy for GA to produce initial population and select the initial searching space, the proposed hybrid algorithm with and without the dynamic adjustment strategy are tested based on IEEE 30-bus system. For the case without dynamic adjustment strategy, when $k=1$ (Step 3), all the initial individuals except one (obtained through rounding off by the solution of IPM from Step 1) are produced randomly within the operational limits of discrete control variables given by (1), and the search area in the whole genetic iteration process is also within this operational limits.

Since the dynamic adjustment strategy mainly affects the process of the first discrete optimization sub-problem solved by GA, only the convergence from the two tests above are compared in Fig. 2, where curves 1 and 2 are the maximum fitness value of population in the iteration process for the case without and with dynamic adjustment strategy, respectively.

For the IEEE 30-bus test system, the active losses are about 0.07 . So the solution may be feasible only when the fitness function given by (8) is around -0.07 .

It is observed from Fig. 2 that obtaining a feasible solution by the hybrid algorithm with and without the dynamic adjustment strategy needs one and ten iterations, respectively. These tests demonstrate that the dynamic adjustment strategy helps to reach a feasible solution with high speed and to improve the efficiency of the hybrid algorithm.

\section{CONCLUSIONS}

The GA is very powerful for dealing with discrete variables, while the IPM is attractive for the efficiency of dealing with the large-scale continuous nonlinear programming. A hybrid method based on GA and IPM has been proposed in this paper. The hybrid method combines the advantages of GA and IPM. A dynamic adjustment strategy has also been proposed to further improve the efficiency of the hybrid method. Numerical simulations on the IEEE 30-bus, IEEE 118-bus and Chongqing 161-bus test systems illustrate that the proposed hybrid algorithm is very efficient.

\section{REFERENCES}

[1] S. S. Sharif and J. H. Taylor, "MINLP formulation of optimal reactive power flow," in Proc. Amer. Control Conf., Albuquerque, NM, 1997, vol. 3, pp. 1974-1978.

[2] J. R. Gomes and O. R. Saaverdra, "Optimal reactive power planning using evolutionary computation: Extended algorithm," Proc. Inst. Elect. Eng., Gen., Transm., Distrib., vol. 146, no. 6, pp. 586-592, Nov. 1999.

[3] K. Y. Lee and F. F. Yang, "Optimal reactive power planning using evolutionary programming: A comparative study for evolutionary programming, evolutionary strategy, genetic algorithm and linear programming," IEEE Trans. Power Syst., vol. 13, no. 1, pp. 101-108, Feb. 1998.

[4] A. G. Bakirtzis, P. N. Biskas, and C. E. Zoumas, "Optimal power flow by enhanced genetic algorithm," IEEE Trans. Power Syst., vol. 17, no. 2, pp. 229-236, May 2002.

[5] S. Granville, "Optimal reactive dispatch through interior point methods," IEEE Trans. Power Syst., vol. 9, no. 1, pp. 136-146, Feb. 1994.

[6] R. A. Jabr, A. H. Coonick, and B. J. Cory, "A primal-dual interior point method for optimal power flow dispatching," IEEE Trans. Power Syst., vol. 17, no. 3, pp. 654-662, Aug. 2002.

[7] M. Liu, S. K. Tso, and Y. Cheng, "An extended nonlinear primal-dual interior-point algorithm for reactive-power optimization of large-scale power systems with discrete control variables," IEEE Trans. Power Syst., vol. 17, no. 4, pp. 982-991, Nov. 2002.

[8] J. R. S. Mantovani, S. A. G. Modesto, and A. V. Garcia, "Var planning using genetic algorithm and linear programming," Proc. Inst. Elect. Eng., Gen., Transm., Distrib., vol. 148, no. 3, pp. 257-262, May 2001.

[9] J. R. O. Soto, C. R. R. Domellas, and D. M. Falcao, "Optimal reactive power dispatch using a hybrid formulation," in Proc. Power Tech, Porto, Portugal, 2001, vol. 3, pp. 5-8.

[10] W. Yan, S. Lu, and D. C. Yu, "A novel optimal reactive power dispatch method based on an improved hybrid evolutionary programming technique," IEEE Trans. Power Syst., vol. 19, no. 2, pp. 913-918, May 2004.

[11] [Online]. Available: http://www.pserc.cornell.edu/matpower/.

[12] [Online]. Available: http://www.ie.ncsu.edu/mirage/GAToolBox/ gaot/.

[13] Y.-M. Park, J.-R. Won, and J.-B. Park et al., "Generation expansion planning based on an advanced evolutionary programming," IEEE Trans. Power Syst., vol. 14, no. 1, pp. 299-305, Feb. 1999.

Wei Yan received the Ph.D. degree in electrical engineering from Chongqing University, Chongqing, China, in 1999.

Currently, he is an Associate Professor and Associate Chairman of the Electrical Power Department in the Electrical Engineering College at Chongqing University. His research interests include optimal operation and control in power systems.

Fang Liu was born in Chongqing, China, on December 16, 1979. She received the B.S. and M.S. degrees from the College of Electrical Engineering, University of Chongqing, in 2001 and 2004, respectively.

She is currently with the Electrical Engineering College at Chongqing University.

C. Y. Chung (M'01) received the B.Eng. degree (with First Class Honors) and the Ph.D. degree in electrical engineering from The Hong Kong Polytechnic University, Hong Kong, China, in 1995 and 1999, respectively.

After his Ph.D. graduation, he worked in the Electrical Engineering Department at the University of Alberta, Edmonton, AB, Canada, and Powertech Labs, Inc., Surrey, BC, Canada. Currently, he is an Assistant Professor in the Electrical Engineering Department of The Hong Kong Polytechnic University. His research interests include power system stability/control, computational intelligence applications, and power markets. 
K. P. Wong (M'87-SM'90-F'02) received M.Sc and Ph.D. degrees from the University of Manchester Institute of Science and Technology (UMIST), Manchester, U.K., in 1972 and 1974, respectively. He was awarded the higher doctorate D.Eng. degree by UMIST in 2001.

$\mathrm{He}$ is currently a Chair Professor and is the Head of Department of Electrical Engineering, The Hong Kong Polytechnic University. He has published numerous research papers in power systems and in the applications of artificial intelligence and evolutionary computation to power system planning and opera- tions. His current research interests include evolutionary optimization in power, power market analysis, power system planning and operation in deregulated environment, power quality.

Prof. Wong received three Sir John Madsen Medals (1981, 1982, and 1988) from the Institute of Engineers Australia, the 1999 Outstanding Engineer Award from IEEE Power Chapter Western Australia, and the 2000 IEEE Third Millennium Award. He is a Fellow of IEE, HKIE, and IEAust. 